\title{
In-House Development, Standardization, and Validation of a RT-qPCR Assay for the Detection of SARS-CoV-2 Virus in Ecuador.
}

\section{David De la Torre}

Universidad Central del Ecuador https://orcid.org/0000-0002-8306-9200

\section{Ronny Pibaque}

Research Institute in Biomedicine

\section{Tatiana Veloz}

Research Institute in Biomedicine

\section{Paúl Beltrán}

Institute for Research in Biomedicine: Institut de Recerca Biomedica

Lucy Baldeón ( $\square$ lybaldeon@uce.edu.ec)

Central University of Ecuador https://orcid.org/0000-0002-0447-0136

\section{Short report}

Keywords: in-house, RT-qPCR, SARS-CoV-2, gene E, COVID-19

Posted Date: October 15th, 2020

DOI: https://doi.org/10.21203/rs.3.rs-91429/v1

License: (c) (1) This work is licensed under a Creative Commons Attribution 4.0 International License. Read Full License 


\section{Abstract}

The World Health Organization (WHO) reported about 30 million cases of Severe acute respiratory syndrome coronavirus 2 (SARS-CoV-2) infection, and around 1 million deaths worldwide. Ecuador is the country with the highest mortality rate of confirmed cases in South America where both the poor ability to identify SARS-CoV-2 carriers and shortages in reagent supply have contributed the high infection rate observed. Hence, there is an urgent need to develop, standardize and validate an in-house protocol that cannot only reduces testing costs, but also increase the ability to screen widely the population. Primerprobe sets for the SARS-CoV-2 envelope protein $E$ and the human ribonuclease $P(R P)$ were validated for a duplex quantitative Reverse Transcription Polymerase Chain Reaction (RT-qPCR) for the Coronavirus Disease-19 (COVID-19) detection. Optimal E primers concentration was $400 \mathrm{nM}$ and TaqMan probe 200 $\mathrm{nM}$. The primer efficiency was set at $94.9 \%$ and $\mathrm{R}^{2}$ value at 0.99 , which was comparable to commercial kits. The lower detection limit was found at 15 copies/ $\mu \mathrm{L}$ (50 copies/rx). In comparison to a Loopmediated Isothermal Amplification (LAMP) commercial kit, there was a higher detection rate (30\%) and results were highly reproducible (95\%). We were able to develop a highly sensitive and low-cost duplex inhouse RT-qPCR test for COVID-19 detection comparable to other commercially available kits.

\section{Background}

The current pandemic has tested the ability of governments to respond to such a worldwide outbreak. The World Health Organization (WHO) reported about 30 million cases of infection for the new coronavirus SARS-CoV-2, and more than 900 thousand deaths until September $17^{\text {th }}$. South America is one of the regions where COVID-19 has rapidly spread [1]. WHO reported that Ecuador confirmed nearly 121 thousand cases and approximately 11 thousand deaths until that same date [2]. According to these statistics, Ecuador has been the country with the highest mortality rate of confirmed cases in South America (9.05\%, S.I. Fig. 1).

One of the reasons explaining the high trend of infection in South America, and particularly in Ecuador, could be the poor ability to identify COVID-19 carriers resulting in low contact traceability [3]. This problem is worsened by worldwide shortages in the reagent supply [4], leading to inaccessible costs of detection kits, particularly, RT-qPCR based ones. This creates an urgent need to standardize an in-house protocol that can not only reduces testing costs, but also increase the ability to screen more population. Improving the ability to identify infected persons both symptomatic and asymptomatic is essential for controlling the global pandemic. Successful cases of wide population screening have been observed in other countries such as South Korea, Taiwan, or Singapore to name a few [5-7].

Testing for COVID-19 can be done in many different ways from RT-qPCR, computer tomography scans [8], to immunoassays [9]. Being the first one, the gold standard to test for SARS-CoV-2 presence as it is the most reliable to detect the virus genomes. This method is based on amplification of small sections of the viral RNA that are unique to SARS-CoV-2. Regions coding for viral structures such as the envelope $E$, the nucleocapsid N proteins, and the RNA-dependent RNA polymerase RdRp have been widely used as the 
choice of biomolecular probes to test for the virus [10]. Several detection kits have been developed around these genetic targets showing high efficiency and sensitivity specially the E target as it has the most conserved nucleotide sequence in SARS-CoV-2 [11]. Both the RdRp and $\mathrm{N}$ genetics targets have lately showed a reduction in their sensitivity [12-14].

In this communication, the development, standardization and validation of an in-house RT-PCR assay protocol for SARS-CoV-2 detection is reported. This standardized procedure does not rely on commercial kits and it was based on the protocol reported by Corman et al., from the University of Charité, Germany [11]. We present an in-house protocol which is primarily based on the envelope protein gene $E$ for COVID19 detection. This gene target is highly recommended by the WHO and the Pan American Health Organization (PAHO) as a diagnostic target and it is considered a highly sensitive probe [11,15-17]. The in-house protocol also includes an internal control for quality of genetic material, the human ribonuclease $\mathrm{P}(\mathrm{RP})$. We tested both genes in a duplex set-up. A multiplex reaction was also explored.

\section{Methods}

Viral RNA material used in this study for standardization procedures came from a viral RNA source with known concentration $\left(3.81 \times 10^{5}\right.$ viral copies per $\left.\mu \mathrm{L}\right)$. For comparative analysis, samples were confirmed to be positive at the Research Institute in Biomedicine (INBIOMED) from the Central University of Ecuador which is an authorized center by the Ecuadorian Ministry of Public Health to handle and test COVID-19 related samples. These samples were positive for both the $\mathrm{N}$ and RdRp for SARS-CoV-2 according to a LAMP based kit 'Isopollo' (Monitor ${ }^{\circledR}$, Daegu, Republic of Korea). Sets of primers-probe target sequences for the E and RdRp SARS-CoV-2 genes were obtained from the protocol published by the University of Charité, Germany, and validated by the WHO [11]. Sets of primers and probe for the RP human gene was obtained from the CDC (S.I. Table 1) [18]. These were designed and purchased from Eurofins (MWG operon, Kentucky, USA). GoTaq(R) Probe 1-step RT-qPCR system reagents were purchased from PROMEGA life sciences (Madison, Wisconsin, USA). All reactions were run on a QuantStudioه 5 Real-Time PCR system (Applied Biosystems).

\section{Primer validation through RT-qPCR and gel electrophoresis.}

Reactions were prepared to test the efficiency of the primers and to find the best conditions for the RTqPCR assay. SARS-CoV-2 genes tested for validation were the $E$, and the RdRp along with a human exogenous target gene RP. Validation reactions had a final volume of $20 \mu \mathrm{L}$ using $5 \mu \mathrm{L}$ of ARN sample and $400 \mathrm{nM}$ concentration for all primer sets. Thermal cycling was performed according to the following conditions: RT-step at $45^{\circ} \mathrm{C}$ for $15 \mathrm{~min}$, Taq-activation at $95^{\circ} \mathrm{C}$ for $2 \mathrm{~min}$ and the 40 PCR cycles performed at $95^{\circ} \mathrm{C}$ for $15 \mathrm{~s}$ and a temperature gradient at 56,58 and $60^{\circ} \mathrm{C}$ for $30 \mathrm{~s}$. Reaction products were looked up by $2 \%$ gel electrophoresis. Sequence lengths were obtained from the NCBI database. (NC_045512.2, https://www.ncbi.nlm.nih.gov).

\section{Simplex (E), duplex (E \& RP) and multiplex (E, RdRp \& RP) RT-qPCR.}


Simplex, duplex, and multiplex reactions were explored. Primer concentrations used were those determined to be optimal in the primer validation step (E: 400, RP: 200 and RdRp: $300 \mathrm{nM}$ ) with their probes at half concentration. Conditions used were the same as the validation step with a small change in the annealing temperature set at $58{ }^{\circ} \mathrm{C}$ and thermal cycles were increased to 45 .

\section{Duplex RT-qPCR: Standard curve and primer efficiency}

To obtain the E primers efficiency under the duplex set up, serial dilutions were prepared from the standardization solution in the range from $2^{15}$ to $2^{11}$ copies per $\mu \mathrm{L}$. With the purpose to improve throughput of the sources and reagents available, optimal primers-probe concentrations were used in a half reaction volume $(10 \mu \mathrm{L})$ set-up. Each assay was prepared in triplicates with a half reaction volume and no ultrapure distilled water was added.

\section{Limit of detection (LoD) of duplex RT-qPCR.}

Following the standard curve experiment, serial dilutions were prepared in the range from 30 to 5 copies per $\mu \mathrm{L}$. Each assay was independently done by three different operators and the reactions were prepared in triplicates according to the duplex set up with the half reaction volume.

\section{Duplex RT-qPCR: Comparative sensitivity and performance.}

Valid samples $(n=93)$ were obtained from INBIOMED with positive $(n=39)$ and negative $(n=54)$ results. Each reaction was prepared according to the duplex set up with the half reaction volume.

\section{Results}

Primer-probe sets were validated through RT-qPCR and gel electrophoresis (S.I. Fig. 2). Amplicons were located at the expected positions for the fragment lengths which were $113 \mathrm{bp}, 100 \mathrm{bp}$ and $50 \mathrm{bp}$ for the $\mathrm{E}$, $\mathrm{RdRp}$, and RP respectively. From the amplification plots, cycle threshold (Ct) values were 25.65 for RdRp, 18.40 for $E$ and 28.68 for RP targets.

RT-qPCR performance (Ct change) and primer reagents usage were parameters used to find an optimal primer concentration. For the RP set, a low variation (0.16) in the $\mathrm{Ct}$ was observed when changing RP primer concentrations from $600 \mathrm{nM}$ to $200 \mathrm{nM}$. The latter was then chosen for all the following assays. In the case for the $E$ set, by taking into consideration the reduction of reagents usage, the primers concentration which had one of the lowest $\mathrm{Ct}$ (18.40) was chosen for remaining experiments, namely 400 $\mathrm{nM}$ for both primers. The annealing temperature that yielded the lower $\mathrm{Ct}$ for the $\mathrm{E}$ target gene was at 58 ${ }^{\circ} \mathrm{C}$ (Data not shown).

Upon optimizing reaction conditions, a reduction in the labor and reagents required was attempted by trying to standardize duplex and multiplex assays. From amplification profiles (Fig.1), there was little variation on the fluorescence for the $\mathrm{E}$ target among all assay. This was also reflected on the $\mathrm{Ct}$ change (Figure 1) suggesting that a multiplex and duplex reaction might be reasonable options for COVID testing. 
From the RP target, which was used to assess genetic material quality, similar results were observed. Despite amplification plots for the RdRp gene not being as reproducible as the other two targets, its $\mathrm{Ct}$ values were within a reasonable margin change (Figure 1). Although this was the case at high RNA concentrations, the RdRp target showed poor or no amplification curves at low concentrations (76-13 viral copies per reaction, data not shown).

Following this assay, the LoD as well as the primer efficiency were determined. Prior to the assay set-up, reduction of the reaction mixture was proposed to increase the throughput of reagents available. Hence, these assays were prepared with a half-reaction volume $(10 \mu \mathrm{L})$. 2-fold serial dilutions were prepared, and a standard curve was obtained. The parameters such as primer efficiency $(94.9 \%)$ and $\mathrm{R}^{2}$ value $(0.99)$ were found within the acceptable range for commercial kits (16) (Fig. 2). Lower LoD of the kit was studied by preparing serial dilutions from 30 to 5 copies $\mu \mathrm{L}$ (Fig. 3). RNA concentrations were shown in number of copies per reaction showing a 100\% detection rate at 50 copies $/ \mathrm{rx}(15 \mathrm{copies} / \mu \mathrm{L})$.

Analytical sensitivity was tested against a commercial and available Isopollo kit which is based on LAMP technique. Of the 93 valid samples used in our study, 39 tested positive and 54 negatives according to Isopollo panel test. By using our in-house protocol, the number of positive hits were 51, representing an increase in $30 \%$ of the relative detection rate (Fig. 4). Some of these samples were replicated $(n=35)$, and it showed a $95 \%$ reproducibility confirming the positive results (Data no shown). The missing $5 \%$ represented two samples with a $\mathrm{Ct}>35$.

\section{Discussion}

The importance of RT-qPCR optimization lies in that unoptimized conditions might give false-positive results as signal might be produced from by non-specific product formation (19). Byproducts, such as longer or shorter amplicons associated respectively with overlapping sequences or primer dimer formation, were not observed in the gels in our experiments, suggesting correct design and high specificity of these primer sets [20-21].

Some attempts have been made to develop a cost-effective kits and to reduce labor time for COVID detection by exploring the efficiency and sensitivity of duplex or multiplex protocols $[19,22]$. This has been done in order to overcome reagent shortages and provide faster results. From the amplification plots, a low variability of our main gen target E was observed in the simplex, duplex and multiplex reactions (Fig. 1). Our method aimed to detect SARS-CoV-2 coronavirus primarily from the $\mathrm{E}$ target gene and counter tested using the RdRp target gene. The usage of the RP gene was proposed to serve as an internal control for sample integrity as recommended by the Centre for Disease Control and Prevention from the United States (CDC) [18]. Although RdRp gene target seemed to work fine at high viral load, its reproducibility and efficiency dropped significantly at low concentrations. Similar findings at low concentrations were observed in other independent studies[12-14]. In our study, we found similar primer efficiency and $\mathrm{R}^{2}$ as those reported by Corman et al., as showed in the standard curve plot (Fig. 2). A similar value for the y-intercept was also found. 
While our in-house protocol was optimized to detect $100 \%$ of SARS-CoV-2 presence up to 50 copies per reaction (Fig. 3), Corman suggested a LoD in the range of 3.9 copies per reaction at $95 \%$ probability from his protocol [11]. A similar reduction in the LoD (276 copies per reaction) was observed in another study which explored the performance of the Cobas ${ }^{\circledR} 6800$ system when employing Cormans' methodology [23]. From the confidence interval obtained in the triplicate reactions, further optimization can be performed (Fig. 3). An excellent starting point would be an exploratory study on selection of RT-qPCR mixes which has been shown to significantly alter the sensitivity of a COVID diagnostic [17].

The higher sensibility from the E gene in a normal RT-qPCR is expected because samples can be considered positive up to a $\mathrm{Ct} \sim 40[11,16]$. Unlikely, other genes in a RT LAMP where there is evidence that a positive sample can be only up to a $\mathrm{Ct} \sim 30$ [24]. In fig. 4, it can be observed that more positive samples were detected by our in-house protocol. One possible explanation for this was found in an independent report which suggested that Isopollo kit possessed a low sensitivity below 1000 copies viral copies per reaction resulting in a lower detection efficiency [25]. It can also be observed a wider $\mathrm{Ct}$ distribution for the E target compared to RdRp from the Isopollo kit. At this stage, it is important to mention that samples were detected positive with the Isopollo kit approximately 1 to 2 months previous to this study. Samples might have suffered couple of freeze-thaw cycles which is known to affect RNA quality. Nonetheless, we observed a $95 \%$ agreement between positive samples (37 out of 39 ), a $30 \%$ higher efficiency in detection of positive cases (51 out of 93) and a 95\% reproducibility (33 out of 35). This two samples possessed a $\mathrm{Ct}>35$, suggesting that repeatability beyond this cycle is not optimal. A couple of extra freeze-thaw cycles might explain this variability.

The duplex set-up reduced reagents usage as well as processing time, while maintaining a high detection rate, modest analytical sensitivity and a much lower cost. Even though the test still has room for improvement, it showed a high sensitivity which is well within the parameters for diagnostics and comparable to other commercial kits [16-17]. Many in-house tests can be found in literature, however, to our knowledge their viability has not been explored in Ecuador. A country where a test at the lowest cost available in the market can represent up to $10 \%$ of the minimum monthly salary. At the Research Institute in Biomedicine, we have standardized a COVID-19 detection workflow from which the value per determination could be capped at less than $2.5 \%$ the minimum wage or less depending on funding available for disposables, reagents and labor.

\section{Conclusion}

We were able to develop, standardize and validate a low-cost duplex in-house RT-qPCR test for COVID detection that can detect $100 \%$ of SARS-CoV-2 presence up to 50 copies per reaction. This matches the efficiency of other commercially available test kits.

\section{Abbreviations}


WHO: World Health Organization. SARS-CoV-2: Severe acute respiratory syndrome coronavirus 2. Ct: Cycle threshold. RT-qPCR: Quantitative reverse transcription polymerase chain reaction. COVID-19: Coronavirus disease 2019. RNA: Ribonucleic acid. PAHO: Pan American Health Organization. LAMP: Loop-mediated isothermal amplification. LoD: Limit of detection. CDC: Centre for Disease Control and Prevention from the United States

\section{Declarations}

\section{Author's contributions}

DT, LB, conceived the study. RP, TV, PB, conducted the research. RP, DT prepared the manuscript. DT, LB, edited the manuscript. All authors read and approved the latest version of the manuscript.

\section{Funding}

This study was co-funded by the Académie De Recherche Et D'Enseignement Supérieur - Universidad Central del Ecuador (ARES-UCE) and La Empresa Pública de Bienes y Servicios -Universidad Central del Ecuador (EP-UCE).

\section{Acknowledgement}

We deeply appreciate Dr. Fernando Sempértegui PhD, Chancellor of the Universidad Central del Ecuador, for his support in managing the needed funds for the development of this in-house RT-qPCR protocol that would have a great positive impact in our society.

\section{Availability of data and materials}

Sequence lengths for the $\mathrm{E}, \mathrm{RdRp}$, and RP genetic targets were obtained from the NCBI database (NC_045512.2, https://www.ncbi.nlm.nih.gov).

\section{Ethics approval and consent to participate}

Not applicable.

\section{Consent to publication}

Not applicable.

\section{Competing interest}

The authors declare that they have no competing interests.

\section{References}


1. Cardona-Ospina JA, Arteaga-Livias K, Villamil-Gómez WE, Pérez-Díaz CE, Katterine Bonilla-Aldana D, Mondragon-Cardona Á, et al. Dengue and COVID-19, overlapping epidemics? An analysis from Colombia. J Med Virol. 2020 Jul 11;1-6.

2. World Health Organization. WHO Coronavirus Disease (COVID-19) Dashboard. [Internet]. [cited 2020 Sep 17]. Available from: https://covid19.who.int/

3. Lima E, Vilela E, Peralta A, Rocha MG, Queiroz BL, Gonzaga MR, et al. Exploring excess of deaths in the context of covid pandemic in selected countries of Latin America. Open Science Framework; 2020 Jun.

4. Guan D, Wang D, Hallegatte S, Davis SJ, Huo J, Li S, et al. Global supply-chain effects of COVID-19 control measures. Nat Hum Behav. 2020 Jun;4(6):577-87.

5. Walensky RP, del Rio C. From Mitigation to Containment of the COVID-19 Pandemic: Putting the SARS-CoV-2 Genie Back in the Bottle. JAMA. 2020 May 19;323(19):1889.

6. Wang CJ, Ng CY, Brook RH. Response to COVID-19 in Taiwan: Big Data Analytics, New Technology, and Proactive Testing. JAMA. 2020 Apr 14;323(14):1341.

7. Lee VJ, Chiew CJ, Khong WX. Interrupting transmission of COVID-19: lessons from containment efforts in Singapore. J Travel Med. 2020 May 18;27(3):1-5.

8. Li W, Cui H, Li K, Fang Y, Li S. Chest computed tomography in children with COVID-19 respiratory infection. Pediatr Radiol. 2020 May;50(6):796-9.

9. Tré-Hardy M, Wilmet A, Beukinga I, Favresse J, Dogné J, Douxfils J, et al. Analytical and clinical validation of an ELISA for specific SARS-CoV-2 IgG, IgA, and IgM antibodies. J Med Virol. 2020 Jul 27;1-9.

10. Atzrodt CL, Maknojia I, McCarthy RDP, Oldfield TM, Po J, Ta KTL, et al. A Guide to COVID-19: a global pandemic caused by the novel coronavirus SARS-CoV-2. FEBS J. 2020 Sep;287(17):3633-50.

11. Corman VM, Landt O, Kaiser M, Molenkamp R, Meijer A, Chu DK, et al. Detection of 2019 novel coronavirus (2019-nCoV) by real-time RT-PCR. Eurosurveillance [Internet]. 2020 Jan 23 [cited 2020 Sep 22];25(3). Available from: https://www.eurosurveillance.org/content/10.2807/15607917.ES.2020.25.3.2000045

12. Vogels CBF, Brito AF, Wyllie AL, Fauver JR, Ott IM, Kalinich CC, et al. Analytical sensitivity and efficiency comparisons of SARS-CoV-2 RT-qPCR primer-probe sets. Nat Microbiol. 2020 Oct;5(10):1299-305.

13. Nalla AK, Casto AM, Huang M-LW, Perchetti GA, Sampoleo R, Shrestha L, et al. Comparative Performance of SARS-CoV-2 Detection Assays Using Seven Different Primer-Probe Sets and One Assay Kit. J Clin Microbiol. 2020;58(6):6.

14. Kim S, Kim D-M, Lee B. Insufficient Sensitivity of RNA Dependent RNA Polymerase Gene of SARSCoV-2 Viral Genome as Confirmatory Test using Korean COVID-19 Cases. other; 2020 Feb.

15. Pan American Health Organization. Directrices de laboratorio para la detección y diagnóstico de la Infección con el nuevo coronavirus 2019 (2019-nCoV). [Internet]. 2020 Jul [cited 2020 Sep 25]. Available from: https://iris.paho.org/handle/10665.2/52471 
16. van Kasteren PB, van der Veer B, van den Brink S, Wijsman L, de Jonge J, van den Brandt A, et al. Comparison of seven commercial RT-PCR diagnostic kits for COVID-19. J Clin Virol. 2020 Jul;128:104412.

17. Alcoba-Florez J, Gil-Campesino H, Artola DG-M de, González-Montelongo R, Valenzuela-Fernández A, Ciuffreda L, et al. Sensitivity of different RT-qPCR solutions for SARS-CoV-2 detection. Int J Infect Dis. 2020 Oct;99:190-2.

18. Centre for Disease Control and Prevention. CDC 2019-Novel Coronavirus (2019 nCoV) Real-Time RTPCR Diagnostic Panel [Internet]. United States; [cited 2020 Sep 25]. Report No.: I. Available from: https://www.fda.gov/media/134922/download

19. Park M, Won J, Choi BY, Lee CJ. Optimization of primer sets and detection protocols for SARS-CoV-2 of coronavirus disease 2019 (COVID-19) using PCR and real-time PCR. Exp Mol Med. 2020 Jun;52(6):963-77.

20. Kanagawa T. Bias and artifacts in multitemplate polymerase chain reactions (PCR). J Biosci Bioeng. 2003 Jan 1;96(4):317-23.

21. Poritz MA, Ririe KM. Getting Things Backwards to Prevent Primer Dimers. J Mol Diagn. 2014 Mar;16(2):159-62.

22. Wang $Y$, Lee $Y$, Yang T, Sun J, Shen C, Cheng C. Current diagnostic tools for coronaviruses-From laboratory diagnosis to POC diagnosis for COVID -19. Bioeng Transl Med. 2020 Sep;5(3):1-10.

23. Pfefferle S, Reucher S, Nörz D, Lütgehetmann M. Evaluation of a quantitative RT-PCR assay for the detection of the emerging coronavirus SARS-CoV-2 using a high throughput system. Eurosurveillance. 2020 Mar 5;25(9):1-5.

24. Dao Thi VL, Herbst K, Boerner K, Meurer M, Kremer LP, Kirrmaier D, et al. A colorimetric RT-LAMP assay and LAMP-sequencing for detecting SARS-CoV-2 RNA in clinical samples. Sci Transl Med. 2020 Aug 12;12(556):1-13.

25. Subsecretaría de Prevención y Promoción de la Salud. Informe de Evaluación Comparativo Preliminar [Internet]. Mexico: Secretaria de Salud; [cited 2020 Sep 25]. Report No.: 1. Available from: https://quito.gob.ec/pruebas/Informe_Mexico.pdf

\section{Figures}



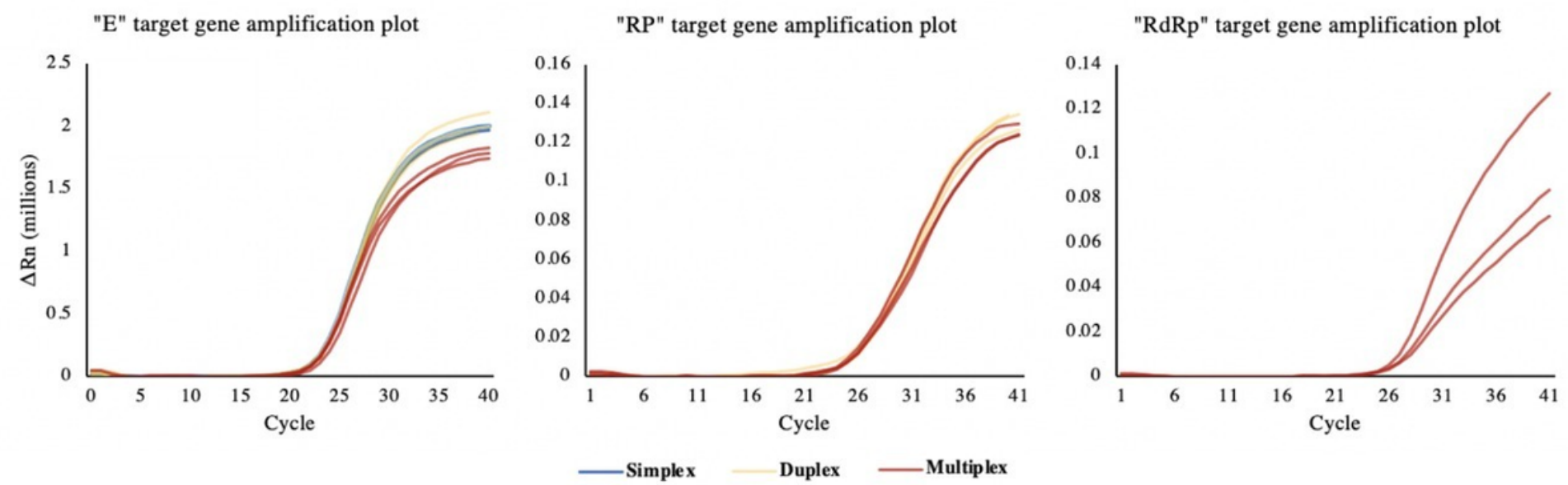

\section{Figure 1}

Amplification plots for the simplex, duplex and multiplex reactions of the three different genes used in this study. Our main detection probe was not significantly affected by the extra addition of other primerprobe sets. Plots were obtained from a QuantStudio $\mathbb{5}$ Real-Time PCR system with detections of the primer sets (E, RP and RdRp) using FAM, VIC and ROX dyes. Concentrations were optimized at $400 \mathrm{nM}$, $200 \mathrm{nM}$ and $300 \mathrm{nM}$ respectively.

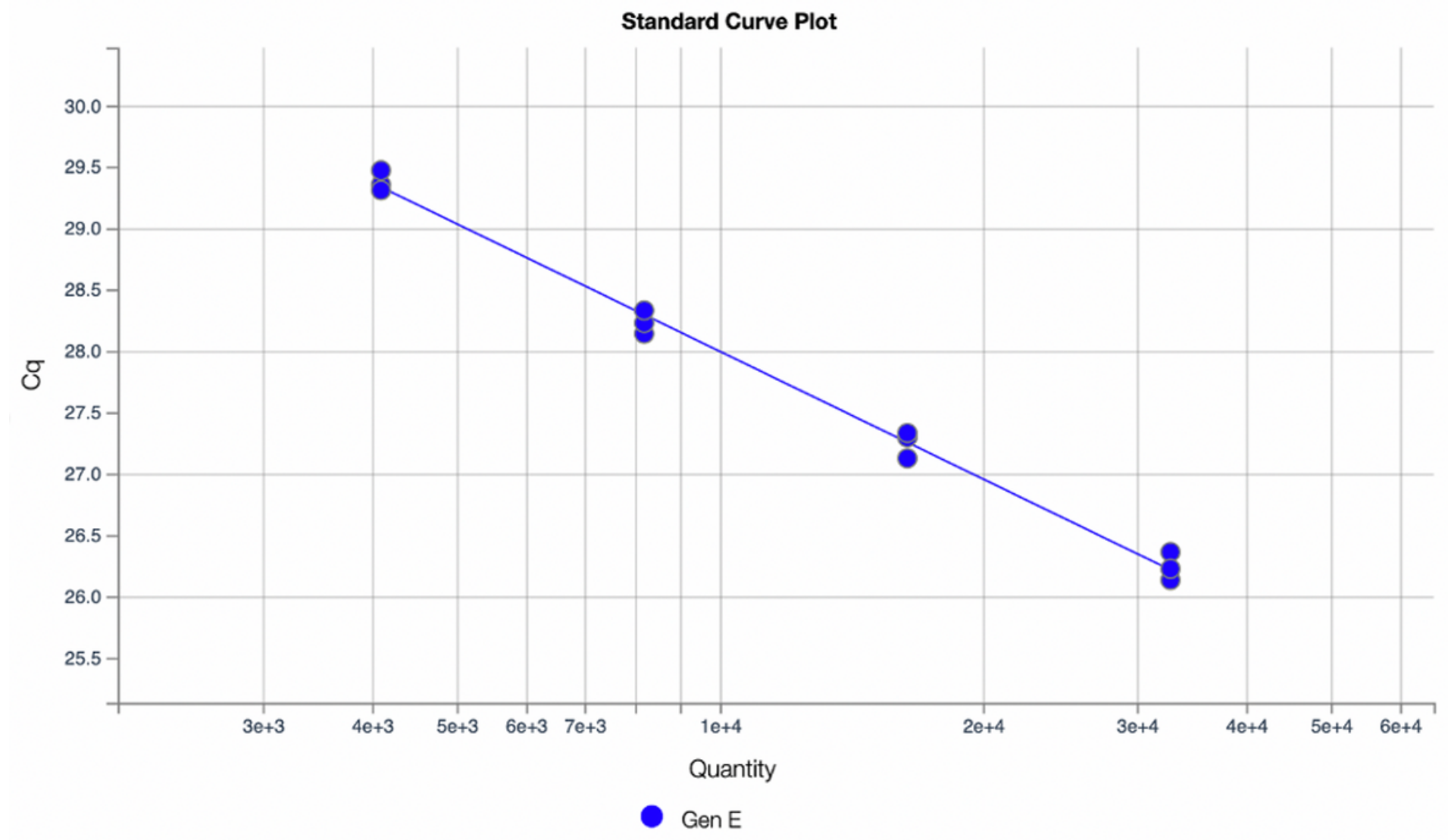

Figure 2 
Standard curve plot of the target gen "E" with R2 $=0.994$, Efficiency $=94.76 \%$, Y-intercept $=41.813$ and slope -3.454. This plot was obtained from 2-fold serial dilutions of standard sample at the range $215-$ 211 copies per $\mu \mathrm{L}$. Concentrations of primers-probe sets for RP and E target genes were used according to the duplex set-up and $10 \mu \mathrm{L}$ reaction volume. Plot was obtained from a QuantStudio 5 Real-Time PCR system.

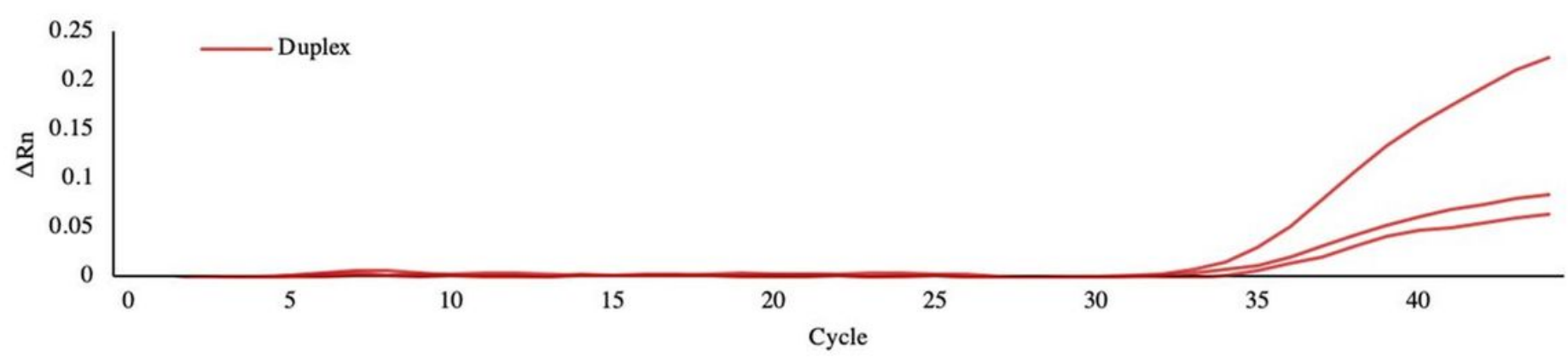

\section{Figure 3}

Amplification plot of the $\mathrm{E}$ target gene at 50 viral copies/rx (15 copies $/ \mu \mathrm{L})$. The average $\mathrm{Ct}$ values was $38.47+/-2.02$ at a $95 \%$ confidence. Amplification plot was obtained from a QuantStudio $\mathbf{5}$ Real-Time PCR system using the duplex set-up. 
$\mathrm{E}$

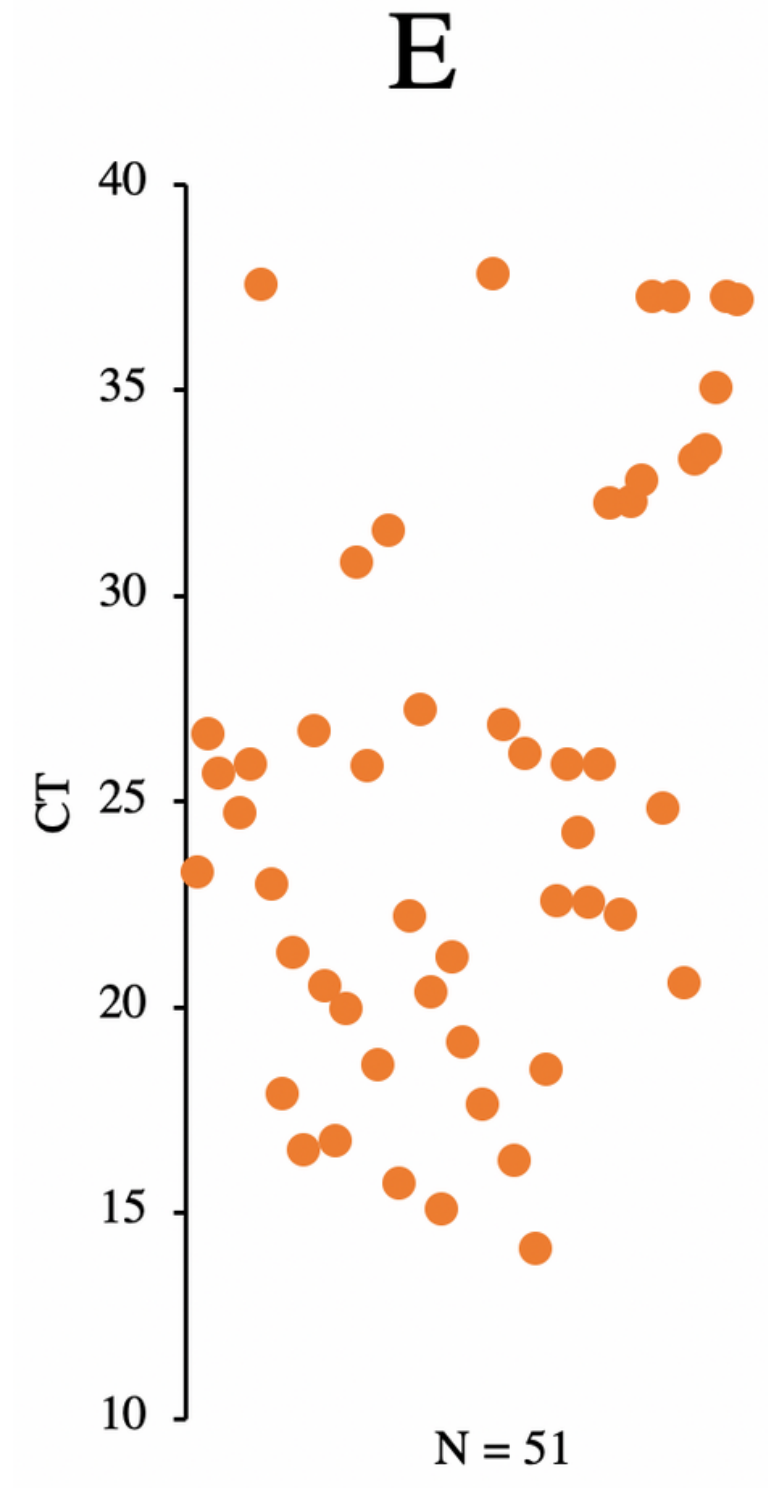

\section{$\operatorname{RdRp}$}

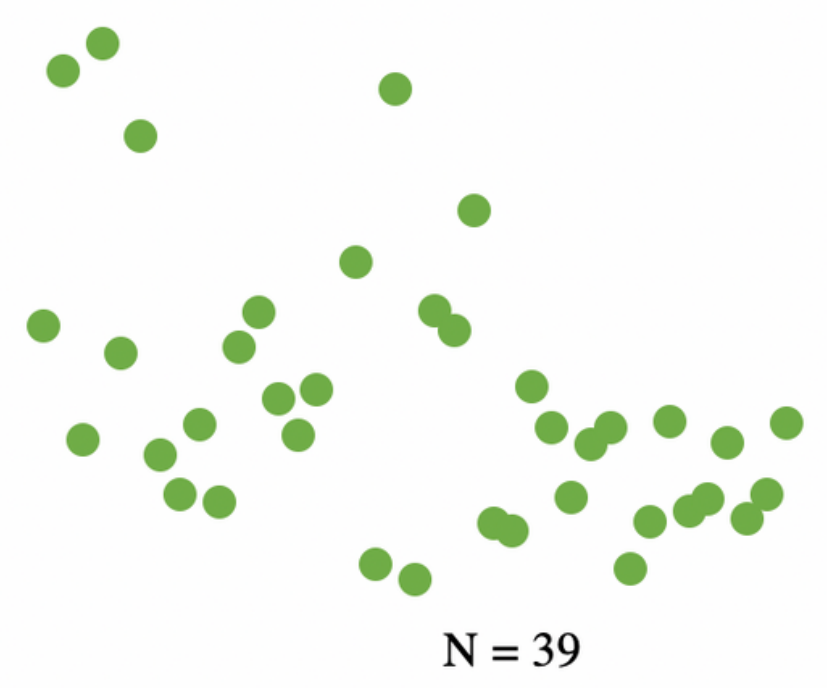

Figure 4

Observed Ct for 93 samples randomly chosen to compare the detection rate of Isopollo RT LAMP kit (RdRp target gene only 'green dots') vs in-house kit (E target gene only 'orange dots'). Reactions for the Isopollo kit were performed according to the manufacturer sheet. In-house protocol reactions were performed according to the duplex set-up.

\section{Supplementary Files}

This is a list of supplementary files associated with this preprint. Click to download.

- supinformation.docx 